\title{
Deskriptive Untersuchung der stationären Aufenthalte von Patienten mit emotional instabiler Persönlich- keitsstörung in Österreich zwischen 2001 und 2016 mit besonderer Berücksichtigung von Alter, Geschlecht und Aufenthaltsdauer
}

\author{
Marlene Koch (D) - Benjamin Vyssoki · Andreas Wippel · Andrea Gmeiner · Nathalie Pruckner · \\ Raimund Oberndorfer
}

Eingegangen: 1. März 2019 / Angenommen: 16. März 2019 / Online publiziert: 2. April 2019

(C) Der/die Autor(en) 2019

\begin{abstract}
Zusammenfassung
Hintergrund Weltweit und insbesondere im stationärpsychiatrischen Setting weist die emotional instabile Persönlichkeitsstörung (EIPS) hohe Prävalenzen auf, die sich hinsichtlich Alter und Geschlecht unterscheiden. Aufgrund des komplexen Krankheitsbildes, wiederholten Aufnahmen und hohen Kosten, wird das Gesundheitssystem vor große Herausforderungen gestellt.

Methoden Für die Berechnungen wurden Statistik Austria-Daten der stationären Aufenthalte von Patienten mit psychischen und Verhaltensstörungen unter besonderer Berücksichtigung der EIPS als Hauptentlassungsdiagnose in Österreich im Zeitraum von 2001-2016 verwendet. Eingeschlossen wurden Patienten mit einem Alter über 14 Jahre, Aufnahme an einer psychiatrischen Klinik in der Akutversorgung und maximale Aufenthaltsdauer von 365 Tagen. Alter, Geschlecht und Aufenthaltsdauer wurden mit SPSS ${ }^{\circledR}$ analysiert, in deskriptiver Form ausgewertet und zueinander in Bezug gesetzt.
\end{abstract}

\footnotetext{
Aus Gründen der besseren Lesbarkeit wurde darauf verzichtet, geschlechtsspezifische Formulierungen zu verwenden. Soweit personenbezogene Bezeichnungen nur in männlicher Form angeführt sind, beziehen sie sich auf Männer und Frauen in gleicher Weise.
}

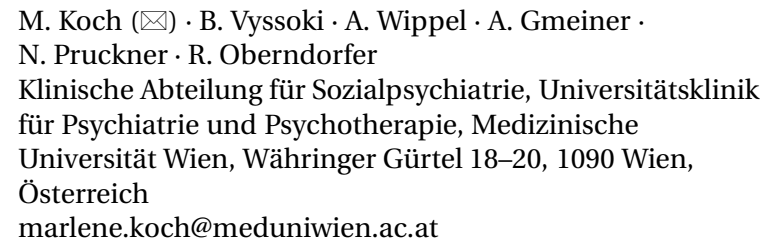

Ergebnisse Die Prävalenz der EIPS innerhalb aller psychischen und Verhaltensstörungen betrug 4,0\%. Von allen Persönlichkeits- und Verhaltensstörungen, sowie spezifischen Persönlichkeitsstörungen wies die EIPS die höchste Prävalenz auf (65,3\% und 79,3\%). Circa viermal mehr Frauen (79,8\%) als Männer (20,2\%) wurden stationär behandelt. Die meisten Patienten $(23,1 \%)$ wurden im Alter von 20-24 Jahren aufgenommen. Ab 40 Jahren zeigte sich die Aufenthaltszahl deutlich rückläufig. Der größte Männeranteil fand sich mit jeweils $27,0 \%$ bei den über 45 -Jährigen und 183-365 Aufenthaltstagen, jener der Frauen zwischen 15-19 Jahren (83,4\%) und 0 bzw. 14-182 Aufenthaltstagen (jeweils 81,4\%). Die mittlere Aufenthaltsdauer betrug für Frauen 13 Tage, für Männer 12 Tage. 11,3\% der Patienten verließen noch am Aufnahmetag die Klinik, 61,2\% wurden 1-13 Tage, 27,2\% 14-182 Tage, und $0,2 \%$ 183-365 Tage behandelt.

Schlüsselwörter Emotional instabile Persönlichkeitsstörung • Borderline Persönlichkeitsstörung · Stationäre Behandlung · Aufenthaltsdauer · Alter · Geschlecht

Descriptive examination of inpatient stays of patients with emotionally unstable personality disorder in Austria between 2001 and 2016 with special consideration of age, gender and length of stay

\section{Summary}

Backround Emotionally unstable personality disorder (EUPD) has a high prevalence worldwide and especially in in-patient psychiatric settings, varying in age 
and gender. Due to the complex clinical picture, repeated admissions and high costs, health systems are facing great challenges.

Methods For the calculations Statistik Austria data of inpatient stays of patients with mental and behavioral disorders with special regard to EUPD as main discharge diagnosis in Austria in the period 2001-2016 were used. Included were patients with age over 14 years, admission to a psychiatric clinic in acute care and maximum length of stay of 365 days. Age, gender and length of stay were analyzed with SPSS $^{\circledR}$, evaluated in descriptive form and related to each other.

Results The prevalence of EUPD within all mental and behavioral disorders was $4.0 \%$. Of all personality and behavioral disorders, as well as specific personality disorders, EUPD had the highest prevalence $(65.3 \%$ and $79.3 \%$ ). About four times more women (79.8\%) than men $(20.2 \%)$ received inpatient treatment. Most patients $(23.1 \%)$ were admitted at the age of $20-24$ years. From 40 years of age the number of admissions clearly decreased. The largest percentage of men was found to be in the age group over 45 years $(27.0 \%)$ and 183-365 inpatient days (27.0\%), those of women aged 15-19 (83.4\%) and 0 and 14-182 inpatient days ( $81.4 \%$ each). The average length of stay was 13 days for women and 12 days for men. $11.3 \%$ of patients left the clinic on the day of admission, $61.2 \%$ were treated for $1-13$ days, $27.2 \%$ for $14-182$ days, and $0.2 \%$ for 183-365 days.

Keywords Emotionally unstable personality disorder · Borderline personality disorder - Inpatient treatment . Length of stay $\cdot$ Age $\cdot$ Sex

\section{Hintergrund}

Die emotional instabile Persönlichkeitsstörung (EIPS, F60.3) zählt im International Statistical Classification of Diseases and Related Health Problems (ICD-10) Klassifikationssystem, Kapitel V, psychische und Verhaltensstörungen (F00-F99) zu den Persönlichkeitsund Verhaltensstörungen (F60-F69) und innerhalb dieser zu den spezifischen Persönlichkeitsstörungen (F60.0-F60.9). Im Gegensatz zu dem in der Literatur zumeist verwendeten Diagnostic and Statistical Manual of Mental Disorders (DSM-5) Klassifikationssystem, wird die EIPS im ICD-10 weiter in einen impulsiven Typ (F60.30) und einen Borderline-Typ (F60.31) differenziert. Diese Unterteilung existiert im DSM-5 nicht, in welchem die „borderline personality disorder" beide Typen der EIPS im ICD-10 umfasst.

Zusätzlich zu den allgemeinen diagnostischen Kriterien von Persönlichkeits- und Verhaltensstörungen ist die EIPS psychopathologisch unter anderem durch eine Instabilität der Affektregulation, Störungen der Identität und der sozialen Interaktionen, Anspannung, Impulsivität, selbstschädigendes Verhalten, vorübergehende dissoziative Symptome, instabile Be- ziehungen, eine hohe Rate an psychischen Komorbiditäten und chronische Suizidalität charakterisiert [1]. Des Weiteren weist die EIPS von allen Persönlichkeitsstörungen mit $7-10 \%$ die höchste Suizidrate auf, die Rate an Suizidversuchen wurde mit circa $60 \%$ beziffert [2].

Die EIPS entwickelt sich meist zwischen Pubertät und jungem Erwachsenenalter und im Verlauf kommt es zu wiederholten Krisen und Rückfällen [3]. Zudem ist die EIPS häufig mit anderen psychiatrischen Komorbiditäten assoziiert. Jene, die für maximal 12 Monate bestehen bleiben, zeigten folgende komorbide Prävalenzen: 50,1\% bipolare Störungen, $51 \%$ Panikattacken mit Agoraphobie, 45,8\% Drogenabhängigkeit, 29,4\% depressive Störungen und 21,5\% Angststörungen. Auch lebenslang bestehende psychiatrische Komorbiditäten weisen erhöhte Prävalenzen auf, wobei hierbei die häufigsten Erkrankungen die Panikstörung mit Agoraphobie (36,0\%) und die bipolare Störung (35,9\%) sind [4].

Weltweit zeigten sich für die EIPS Prävalenzraten von $1-6 \%$ in der Normalbevölkerung, 9-22\% im psychiatrisch-ambulanten und 20-49\% im psychiatrischstationären Setting [1, 5, 6]. Aufgrund der hohen Rate an Komorbiditäten und damit verbundenen Schwierigkeiten bei der korrekten Diagnosestellung variierten die Prävalenzraten mitunter stark [4].

Trotz der weit verbreiteten Annahme, dass vermehrt Frauen an einer EIPS leiden, konnten in den meisten epidemiologischen Untersuchungen keine Unterschiede der Prävalenzraten zwischen Frauen und Männern festgestellt werden [7-9]. Im Gegensatz dazu wurde in klinischen Studien häufig ein höherer Frauenanteil beschrieben, was auf die erhöhte Bereitschaft von Frauen nach Hilfe zu suchen [10], aber auch biologische oder soziokulturelle Unterschiede zurückzuführen sein könnte [11, 12]. Insgesamt zeigten Männer und Frauen mit EIPS in ihrem Denken, Fühlen und Verhalten mehr Gemeinsamkeiten als Unterschiede [12, 13]. Bei Männern lagen jedoch häufiger ein komorbider Substanzmissbrauch, eine schizotype, narzisstische oder antisoziale Persönlichkeitsstörungen vor, während bei Frauen häufiger Essstörungen oder eine PTSD als Komorbiditäten beobachtet wurden [4, 13].

Patienten mit EIPS weisen, vor allem durch wiederholte Nutzung, im Vergleich zu Patienten mit anderen Persönlichkeitsstörungen die höchste Inanspruchnahme psychiatrischer Dienste im öffentlichen Sektor auf [14-16]. Insbesondere stationäre Aufenthalte sind aufgrund der Häufigkeit der mit EIPS verbundenen persönlichen Krisen und Suizidalität keine Seltenheit $[15,16]$.

Im Gegensatz zum früheren Konzept der stets lebenslang bestehenden Persönlichkeitsstörung, zeigten Studien der letzten Jahre, dass innerhalb von 10 Jahren nach stationärer Behandlung bei bis $\mathrm{zu}$ $85 \%$ der Patienten eine mindestens 2 Jahre anhaltende Remission erzielt werden konnte [17, 18]. Rück- 
fallsraten nach einer erreichten Remission zeigten sich innerhalb von 10 Jahren zwischen 10 und $20 \%$ [18]. Laut einer Langzeitstudie von Zanarini et al. aus dem Jahr 2012 erreichten nur 30\% der Patienten eine stabile Genesung inklusive sozialer und beruflicher Integration [17].

Trotz geringer Evidenz für die Wirksamkeit psychopharmakologischer Behandlungsansätze, erhalten Patienten mit EIPS in hohem Ausmaß medikamentöse Behandlungen mit diversen Substanzen (Antipsychotika, Antidepressiva, Benzodiazepine, Antikonvulsiva) [14]. Als Therapie der Wahl mit der besten Studienlage und Effektivität gilt bei Patienten mit EIPS die dialektisch-behaviorale Therapie [19].

Zweck unserer Untersuchung war es, Faktoren wie Alter, Geschlecht und Aufenthaltsdauer von Patienten mit EIPS für den Zeitraum von 2001-2016 zu analysieren, um bestimmte zugrundeliegende Muster im Aufnahmeverhalten auf psychiatrischen Abteilungen in der Akutversorgung zu erheben.

\section{Methoden}

Die Daten wurden von Statistik Austria in anonymisierter Form bereitgestellt. Daten über das österreichische Gesundheitssystem werden jährlich von Statistik Austria gesammelt und der wissenschaftlichen Forschung zur Verfügung gestellt. Es wurden sämtliche von Statistik Austria bereitgestellte Daten bezüglich der stationären Aufenthalte von Patienten mit psychischen- und Verhaltensstörungen (F00-F99) nach ICD10 als Hauptentlassungsdiagnose in Österreich im Zeitraum von 2001 bis 2016 für die Untersuchung berücksichtigt. Nebendiagnosen wurden nicht erhoben und konnten daher nicht zur weiteren Auswertung herangezogen werden. Einschlusskriterien waren Alter über 14 Jahre, Aufnahme an einer psychiatrischen Klinik in der Akutversorgung und eine maximale Aufenthaltsdauer von 365 Tagen. Ausgeschlossen wurden
Daten von Patienten mit einer stationären Aufenthaltsdauer über einem Jahr, Aufnahmen an nichtpsychiatrischen Kliniken und andere Versorgungssektoren außerhalb der Akutversorgung (Genesung/ Prävention, Langzeitversorgung, Rehabilitation). Faktoren wie Geschlecht, Alter und Aufenthaltsdauer wurden mithilfe von SPSS ${ }^{\circledR}$ Software [20] analysiert und in deskriptiver Form ausgewertet und zueinander in Bezug gesetzt.

\section{Ergebnisse}

Nach Ausschluss der zuvor definierten Kriterien verblieben insgesamt 50.644 (89,1\%) von 56.855 Patienten mit EIPS (F60.3) als Hauptentlassungsdiagnose zur weiteren Analyse der Alters- und Geschlechterverteilung und deskriptiven Auswertung.

Die Prävalenz der EIPS bezogen auf die psychischen und Verhaltensstörungen betrug 4,0\% ( $n=50.644$ von 1.264.199). Von allen Persönlichkeits- und Verhaltensstörungen wies die EIPS mit 65,3\% ( $n=50.644$ von 77.577) die höchste Prävalenz auf. In der Gruppe der spezifischen Persönlichkeitsstörungen lag sie mit $79,3 \%$ ( $n=50.644$ von 63.872) sogar noch deutlich höher.

Von insgesamt 50.644 Patienten mit EIPS wurden mit $79,8 \% \quad(n=40.415)$ fast viermal mehr Frauen als Männer $(20,2 \%, n=10.229)$ zwischen 2001 und 2016 in der psychiatrischen Akutversorgung in Österreich stationär behandelt.

Bei beiden Geschlechtern fanden die mit Abstand meisten stationären Aufenthalte mit 23,1\% $(n=11.692)$ im Alter von 20 bis 24 Jahren statt. Ab einem Alter von 24 Jahren zeigte sich die Anzahl der stationären Behandlungen kontinuierlich rückläufig auf bis $\mathrm{zu}$ jeweils 8,6\% in den Altersgruppen der 40-44-Jährigen und der über 45-Jährigen (Abb. 1).

Bezogen auf unterschiedliche Altersgruppen zeigte sich, dass das Verhältnis von circa $80 \%$ Frauen zu $20 \%$

Abb. 1 Altersverteilung der EIPS in Prozent (\%)

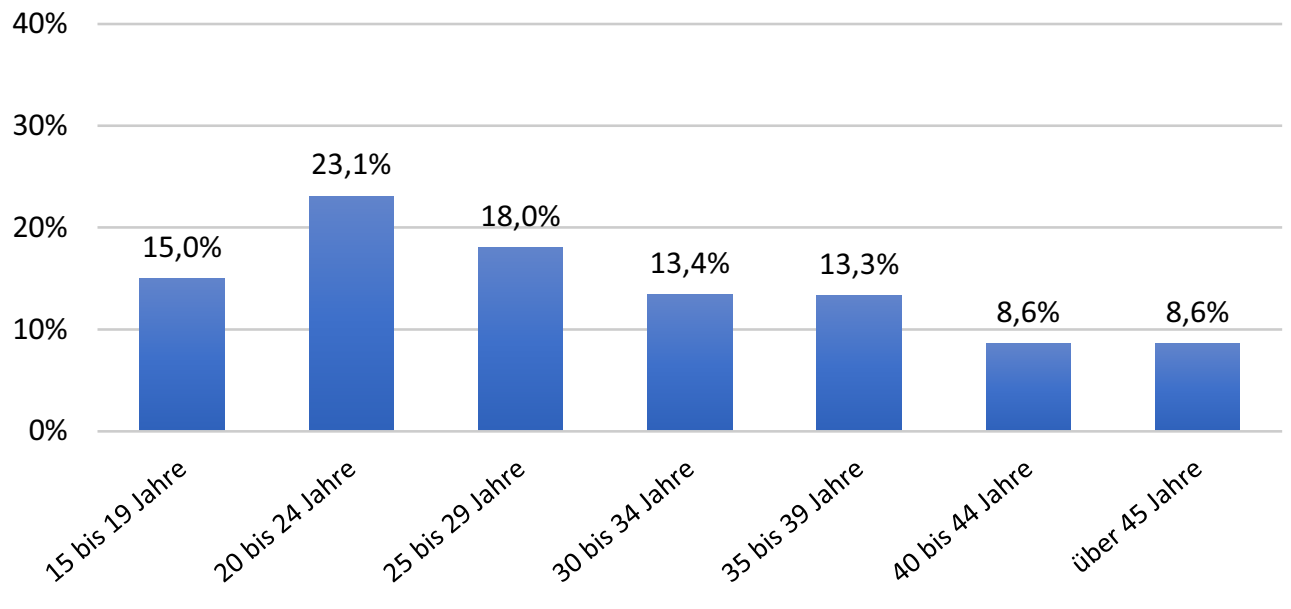


Tab. 1 Geschlechterverteilung von Patienten mit EIPS innerhalb verschiedener Altersgruppen

\begin{tabular}{|l|l|l|l|l|l|l|}
\hline Alter (Jahre) & Männer absolut & Männer in \% & Frauen absolut & Frauen in \% & $\begin{array}{l}\text { Gesamt } \\
\text { absolut }\end{array}$ & Gesamt in \% \\
\hline $15-19$ & 1257 & 16,6 & 6333 & 83,4 & 7590 & 15,0 \\
\hline $20-24$ & 2256 & 19,3 & 9436 & 80,7 & 11692 & 23,1 \\
\hline $25-29$ & 1803 & 19,8 & 7316 & 80,2 & 9119 & 18,0 \\
\hline $30-34$ & 1444 & 21,3 & 5339 & 78,7 & 6783 & 13,4 \\
\hline $35-39$ & 1315 & 19,5 & 5423 & 80,5 & 6738 & 13,3 \\
\hline $40-44$ & 980 & 22,4 & 3395 & 77,6 & 4375 & 8,6 \\
\hline C45 & 1174 & 27,0 & 3173 & 73,0 & 4347 & 8,6 \\
\hline Gesamt & 10.229 & - & 40.415 & - & 50.644 & 100 \\
\hline
\end{tabular}

Männer bis zum Alter von 44 Jahren bestehen blieb. Der mit 83,4\% ( $n=6333)$ höchste Frauenanteil zeigte sich in der Gruppe der 15- bis 19-Jährigen $(n=7590)$. Im Gegensatz dazu erfolgten mit 27,0\% $(n=1174)$ bei den Männern die mit Abstand meisten stationären Aufenthalte ab einem Alter von 45 Jahren $(n=4347)$ (s. Tab. 1).

Die mittlere Aufenthaltsdauer von Patienten mit EIPS an psychiatrischen Abteilungen in der Akutversorgung in Österreich zwischen 2001 und 2016 lag bei 13 Tagen, wobei Frauen im Durchschnitt einen Tag länger als Männer stationär behandelt wurden (13 vs. 12 Tage).

Die mit $61,2 \%(n=31.014)$ meisten Patienten blieben 1-13 Tage in stationärer Behandlung, $13791 \mathrm{~Pa}-$ tienten $(27,2 \%)$ blieben 14-182 Tage. 5717 Patienten $(11,3 \%)$ verließen noch am Tag der Aufnahme die Klinik (0-Tage Aufenthaltsdauer). 122 Patienten (0,2\%) wurden 183-365 Tage an einer psychiatrischen Klinik in der Akutversorgung behandelt (s. Abb. 2).

Während die prozentuale Häufigkeit der Männer mit Aufenthaltsdauer 0 Tage und 14-182 Tage jeweils bei 18,6\% lag, zeigte sich ein mit 21,2\% höherer Männeranteil in der Gruppe der Aufenthaltsdauer von 1-13 Tage. Der größte Anteil an Männern (27,0\%) konnte in der Gruppe der längsten Aufenthaltsdauer von 183-365 Tagen gezeigt werden, jener der Frauen war mit jeweils $81,4 \%$ in den Gruppen der 0 bzw. 14-182 Aufenthaltstage am höchsten (s. Abb. 2).

\section{Diskussion}

Durch die deskriptive Auswertung der von Statistik Austria gesammelten Daten in Bezug auf Alter, Geschlecht und Aufenthaltsdauer von Patienten mit EIPS als Hauptentlassungsdiagnose in Österreich von 2001-2016, konnten wir in mehrerer Hinsicht interessante und relevante Ergebnisse darstellen.

Zusätzlich zu den in der Einleitung genannten internationalen EIPS Prävalenzraten in der Normalbevölkerung, dem psychiatrisch-ambulanten und -stationären Setting, konnten wir für Österreich zwischen 2001-2016 Prävalenzen der EIPS innerhalb aller psychischen und Verhaltensstörungen, aller Persönlichkeits- und Verhaltensstörungen, sowie der spezifischen Persönlichkeitsstörungen errechnen.

Im Vergleich zu den in der Literatur zumeist identifizierten Prävalenzzahlen der EIPS unter stationärpsychiatrischen Patienten von circa $20 \%$ [4], zeigte sich in unserer repräsentativen, gesamt-österreichi-
Abb. 2 Aufenthaltsdauer gruppiert (0 Tage, 1-13 Tage, 14-182 Tage, 183-365 Tage) und aufgeteilt nach Geschlecht in absoluten Zahlen

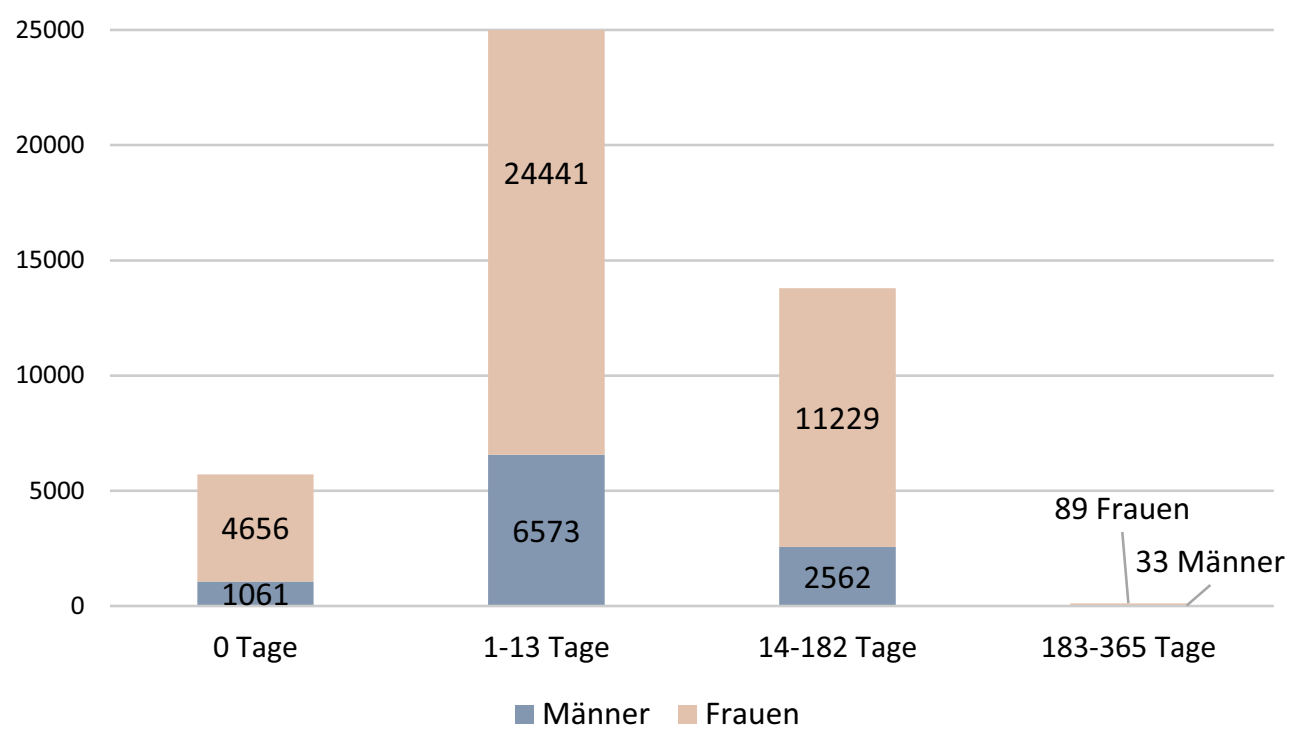


schen Stichprobe der EIPS eine fünffach niedrigere Prävalenz von 4,0\% innerhalb aller Psychischen- und Verhaltensstörungen. Dies könnte unter anderem durch die höhere Fallzahl unserer Studie erklärt werden.

Trotz eines vielfach postulierten, allgemein in etwa ausgeglichenen Geschlechterverhältnisses [7-9] zeigte sich in unserer Stichprobe ein vierfach höherer Frauenanteil unter den in der psychiatrischen Akutversorgung in Österreich aufgenommen Patienten mit EIPS zwischen 2001-2016 (circa 80\% Frauen, 20\% Männer). Diese in der Tendenz bekannte Diskrepanz könnte, wie einleitend bereits beschrieben, unter anderem auf eine bei Frauen erhöhte Inanspruchnahme von therapeutischer Unterstützung [10] oder biologische bzw. soziokulturelle Unterschiede erklärt werden [11, 12].

In Bezug auf das Alter zeigten sich bei beiden Geschlechtern im Alter von 20 bis 24 Jahren die mit Abstand meisten stationären Aufnahmen ( $n=11.692$, 23,1\%), während sich die Anzahl der stationären Behandlungen ab einem Alter von 24 Jahren kontinuierlich rückläufig auf bis zu jeweils $8,6 \%$ in den Altersgruppen der 40-44-Jährigen und der über 45-Jährigen zeigten (Abb. 1).

Der mit 83,4\% höchste Frauenanteil zeigte sich in der Gruppe der 15- bis 19-Jährigen, was mit dem Beginn der Erkrankung in der frühen Adoleszenz zusammenfällt. Hingegen konnten wir bei den Männern die mit 27,0\% mit Abstand häufigsten stationären Aufnahmen in der Altersgruppe der über 45-Jährigen feststellen, was einem relativen Anstieg der Häufigkeit der EIPS bei Männern im Vergleich zu Frauen darstellt und in dieser Form unserer Kenntnis nach erstmalig dargestellt wurde. Zum Vergleich beträgt der Anteil der Männer innerhalb der gesamten EIPS Patientenpopulation dieser Studie 20,2\%.

Wie einleitend bereits beschrieben, weisen Patienten mit EIPS durch wiederholte Nutzung des Gesundheitssystems die höchste Inanspruchnahme psychiatrischer Dienste im öffentlichen Sektor auf [14-16]. Patienten mit EIPS zeigten eine 2- bis 3-mal höhere Wahrscheinlichkeit der Wiederaufnahme nach Entlassung aus einem stationären Setting, im Vergleich zu anderen Persönlichkeitsstörungen [21]. Gleichzeitig wurden stationäre Aufenthalte von Patienten mit EIPS von einigen Autoren als sowohl ineffektiv, als auch kontraproduktiv angesehen. Während manche Autoren die Meinung vertreten Patienten mit EIPS am besten überhaupt nicht in ein stationäres Setting aufzunehmen [22], sehen andere Autoren die Wichtigkeit darin, den klinischen Aufenthalt zumindest möglichst kurz zu halten [16]. Vor diesem kontroversiellen Hintergrund gewinnt es an Bedeutung, dass sich die Patienten unserer Stichprobe durchschnittlich 13 Tage lang in stationärer Behandlung befanden. In Bezug auf die Geschlechterverteilung zeigte sich jedoch, trotz des deutlich höheren Frauenanteils von circa $80 \%$, eine im Durchschnitt nur um einen Tag längere stationäre Aufenthaltsdauer von Frauen im Vergleich zu
Männern (13 vs. 12 Tage). Die kürzeste Aufenthaltsdauer betrug 0 Tage, die längste Aufenthaltsdauer 364 Tage. Hierbei ist zu berücksichtigen, dass Daten von Patienten mit einer Aufenthaltsdauer über einem Jahr zur besseren Darstellung der Ergebnisse und zur Vermeidung von extremen Einzelwerten ausgeschlossen wurden.

Nachdem die unterschiedlichen stationären Verweildauern geschlechtsspezifisch differenziert zusammengefasst wurden, zeigte sich, dass mit $61,2 \%$ die Mehrheit der Patienten zwischen 1 und 13 Tagen behandelt wurden. 11,3\% wiesen eine Aufenthaltsdauer von 0 Tagen auf, wobei hier anzunehmen ist, dass jene Patienten bereits am Aufnahmetag wieder das Krankenhaus verließen, wofür die mit der EIPS störungsspezifisch-assoziierte Ambivalenz, Impulsivität und Labilität der Patienten mitverantwortlich gewesen sein könnte. 27,2\% der Patienten befanden sich zwischen 2 Wochen und 6 Monaten, und $122 \mathrm{~Pa}$ tienten $(0,2 \%)$ darüber hinaus sogar bis zu einem Jahr in der psychiatrischen Akutversorgung in stationärer Behandlung (s. Abb. 2).

Vergleicht man sowohl die Verteilung der Geschlechter innerhalb der verschiedenen Altersgruppen (s. Tab. 1 und Abb. 1), als auch die Verteilung der Geschlechter innerhalb der AufenthaltsdauerGruppen (s. Abb. 2), zeigt sich ein annähernd gleichbleibendes Verhältnis von viermal mehr Frauen (circa $80 \%$ ) als Männer (circa 20\%): in der Gruppe der Aufenthaltsdauer 0 und $14-182$ Tage je $81,4 \%$, in der Gruppe der Aufenthaltsdauer 1-13 Tage 78,8\%.

Des Weiteren zeigte sich bei den Männern sowohl in der Gruppe der über 45-Jährigen, als auch in jener mit der längsten Aufenthaltsdauer (6-12 Monate) ein Anstieg von circa $20 \%$ auf $27,0 \%$ (s. Tab. 1 und Abb. 2). Als Erklärung hierfür könnte ein sich mit zunehmendem Alter und vermehrten beziehungsweise chronifizierten Komorbiditäten, wie zum Beispiel Suchterkrankungen, komplizierter bzw. komplikationsreicher Krankheitsverlauf ebenso herangezogen werden, wie eine im Verlauf höhere Behandlungsbereitschaft von Männern oder eine gestiegene Remissionsrate bei Frauen. Andererseits kommt weitergedacht jedoch auch ein Versorgungsmangel im nichtakutpsychiatrischen Behandlungs- und Betreuungssektor infrage.

Einschränkend muss festgehalten werden, dass es durch die Verwendung anonymisierter Daten nicht möglich war, Patienten individuell zu erfassen und dadurch keine Aussagen über die Gesamtzahl aller stationären Aufenthalte pro Patienten oder etwaige Komorbiditäten getroffen werden konnten. Des Weiteren bringt die Verwendung von Nutzungsdaten Limitationen, wie zum Beispiel Fehlklassifizierungen (durch nicht nachvollziehbare Diagnostik) mit sich, welche zu Diskrepanzen zwischen unseren Zahlen und jener anderer Untersuchungen beitragen können.

Des Weiteren ist wie einleitend erwähnt zu berücksichtigen, dass im ICD-10 die EIPS in einen impul- 
siven Typ und einen Borderline Typ untergliedert ist. Im für wissenschaftliche Zwecke gängigeren DSM-5 ist diese Unterteilung jedoch nicht vorhanden, wohingegen hier die EIPS übergreifend als „Borderline personality disorder" bezeichnet wird. Aufgrund der uns zur Verfügung gestandenen Daten mit auf vier Stellen begrenztem ICD-Diagnose-Code (F60.3) war es nicht möglich eine Differenzierung der Typen vorzunehmen.

\section{Fazit für die Praxis}

- Vor dem Hintergrund einer hohen Prävalenz und Suizidrate, eines hohen Leidensdrucks, komplizierter Verläufe mit zahlreichen Komorbiditäten und häufigen Rückfällen mit insgesamt hochfrequenter Nutzung des Gesundheitssystems und damit assoziiert hohen Kosten, erscheint eine flächendeckende, möglichst frühzeitige und niederschwellige Verfügbarkeit von sowohl finanziell als auch fachlich ausreichend gut ausgestatteten Versorgungsstrukturen zur Diagnostik und Therapie von Patienten mit EIPS dringend notwendig.

- Unter anderem in Anbetracht der von uns dargestellten Diskrepanz zwischen evidenzbasierten Empfehlungen - möglichst kurze stationäre Aufenthaltsdauer und ambulante dialektisch-behaviorale Therapie als effektivste Therapie - einerseits, und einer mittleren Aufenthaltsdauer von 13 Tagen in Österreich andererseits, wobei über ein Viertel $(27,4 \%)$ aller Aufenthalte zwischen 2 Wochen und einem Jahr betrugen, lässt sich auf einen großen Aufholbedarf des öffentlichen und insbesondere des psychiatrisch-psychotherapeutischen Gesundheitswesens und seiner Strukturen in Österreich schließen.

- Letztlich ist eine grundlegende, differenzierte und aussagekräftige statistische Erfassung und Auswertung quantitativ und qualitativ hochwertiger Daten zum Status quo der bestehenden Versorgungsstrukturen eine unerlässliche Voraussetzung für eine erfolgreiche, weitreichendere Planung und Durchführung gezielter Therapieinterventionen. Weitergehende Untersuchungen auf verschiedenen Ebenen, so etwa der regional verfügbaren störungsspezifischen Therapieangebote, sind zur Beurteilung und Verbesserung der Versorgungssituation essentiell.

Funding Open access funding provided by Medical University of Vienna.

Interessenkonflikt M. Koch, B. Vyssoki, A. Wippel, A. Gmeiner, N. Pruckner und R. Oberndorfer geben an, dass kein Interessenkonflikt besteht.

Open Access Dieser Artikel wird unter der Creative Commons Namensnennung 4.0 International Lizenz (http:// creativecommons.org/licenses/by/4.0/deed.de) veröffentlicht, welche die Nutzung, Vervielfältigung, Bearbeitung, Verbreitung und Wiedergabe in jeglichem Medium und Format erlaubt, sofern Sie den/die ursprünglichen Autor(en) und die Quelle ordnungsgemäß nennen, einen Link zur Creative Commons Lizenz beifügen und angeben, ob Änderungen vorgenommen wurden.

\section{Literatur}

1. Lieb K, Zanarini M, Schmahl C, Linehand M, Bohus M. Borderline personality disorder. Lancet. 2004;364:453-61.

2. Bohus M, Remmel A. Zum Umgang mit Suizidalität in der Borderline-Therapie. Persönlichkeitsstörungen Theor Ther. 2004;8:11-6.

3. Winograd G, Cohen P, Henian C. Adolescent borderline symptoms in the community: prognosis for functioning over 20 years. J Child Psychol Psychiatry. 2008;49:933-41.

4. Grant B, Chou S, Goldstein R, Huang B, Stinson F, Saha T, Smith S, Dawson D, Pulay A, Picerking R, Ruan W. Prevalence, correlates, disability, and comorbiditiy of DSM IV borderline personality disorder: results from the wave 2 national epidemiologic survey on alcohol and related conditions. JClin Psychiatry. 2008;69:533-45.

5. Ellison W, Rosenstein L, Morgan T, Zimmerman M. Community and clinical epidemiology of borderline personality disorder. Psychiatr Clin North Am. 2018;41(4):561-73.

6. Chanen A, McCutcheon L. Prevention and early intervention for borderline personality disorder: current status and recent evidence. Br J Psychiatry Suppl. 2013;54:24-9.

7. Coid J, Yang M, Tyrer P, Roberts A, Ullrich S. Prevalence and correlates of personality disorder in Great Britain. Br J Psychiatry. 2006;188:423-31.

8. TorgersenS, KringlenE,CramerV.Theprevalenceofpersonality disorders in a community sample. Arch Gen Psychiatry. 2001;58(6):590-6.

9. Jackson H, Burgess P. Personality disorders in a community: a report from the Australian National Survey of Mental Health and Well-Being. Soc Psychiatry Psychiatr Epidemiol. 2000;35:531-88.

10. Bohus M, Kröger C. Psychopathology and psychotherapy of borderline personality disorder: state of the art. Nervenarzt. 2011;82(1):16-24.

11. Zlotnick C, Rothschild L, Zimmerman M. The role of gender in the clinical presentation of patients with borderline personality disorder. JPersonal Disord. 2002;16:277-82.

12. Skodal A, Bender D. Why are women diagnosed borderline more than men? Psychiatr Q. 2003;74:349-60.

13. Johnson D, Shea M, Yen S, Battle C, Zlotnick C, Sanislow C, Grilo C, Skodol A, Bender D, McGlashan T, Gunderson J, Zanarini M. Gender differences in borderline personality disorder:findings from theCollaborative Longitudinal Personality Disorders Study. Compr Psychiatry. 2003;44(4):284-92.

14. Zanarini M, Frankenburg F, Hennen J, Silk K. Mental health service utilization by borderline personality disorder patients and axis II comparison subjects followed prospectively for 6 Years. J Clin Psychiatry. 2004;65:28-36.

15. Bender D, Dolan R, Skodol A, Sanislow C, Dyck I, McGlashan T, Shea M, Zanarini M, Oldham J, Gunderson J. Treatment utilisation by patients with personality disorders. Am J Psychiatry. 2001;158(2):295-302.

16. Linehan M, Heard H. Borderline personality disorder: costs, course, and treatment outcomes. In: Miller N, Magruder $\mathrm{K}$, Hrsg. The cost effectiveness of psychotherapy: a guide for practitioners. New York: Oxford University Press; 1999. S.291-305.

17. Zanarini M, Frankenburg F, Reich D, Fitzmaurice G. Attainment and stability of sustained symptomatic remission and recovery among borderline patients and axis II compari- 
son subjects: a 16-year prospective follow-up study. Am J Psychiatry. 2012;169(5):476-83.

18. Sanislow C, Marcus K, Reagan E. Long-term outcomes in borderline psychopathology: old assumptions, current findings, and new directions. Curr Psychiatry Rep. 2012;14(1):54-61.

19. Comtois A, Carmel A. Borderline personality disorder and high utilization of inpatient psychiatric hospitalization: concordance between research an clinical diagnosis. J BehavHealth Serv Res. 2016;43(2):272-80.

20. IBM Corp. IBM SPSS Statistics for Windows, Version 25.0. Armonk:IBMCorp; 2017.
21. Lewis K, Fanaian M, Kotze B, Grenyer B. Mental health presentations to acute psychiatric services: 3-year study of prevalence and readmission risk for personality disorders compared with psychotic affective, substance or other disorders. BJPsych Open. 2019;1:1-7.

22. Dawson D, MacMillan H. Relationship management of the borderline patient: from understanding to treatment. New York: Brunner/Mazel; 1993.

Hinweis des Verlags Der Verlag bleibt in Hinblick auf geografische Zuordnungen und Gebietsbezeichnungen in veröffentlichten Karten und Institutsadressen neutral. 